\title{
Hacia un reposicionamiento de la investigación cualitativa en Tecnología Educativa
}

\author{
Isabel Mª Solano Fernández \\ Universidad de Murcia \\ imsolano@um.es
}

La investigación en Tecnología Educativa ha tenido una gran evolución en las últimas décadas, encontrándose una importante producción en revistas especializadas y no especializadas (Valverde, 2016). Aún así llama la atención el "cierto retroceso de sus principios, que afortunadamente se está superando, por su equiparación desde ciertos sectores con la aplicación de las Tecnologías de la Información y Comunicación (TIC)" (Cabero, 2016, p. 23).

La investigación en este campo de conocimiento se ha movido en dos grandes visiones: La aplicación de los medios y el diseño de la instrucción (Cabero, 2016). A pesar de ello, recientemente se ha comenzado a abordar desde una perspectiva más holística, que permite analizar estos medios, evaluarnos o aplicarlos desde un contexto formal, no formal e informal. Ampliamos así el ámbito de estudio de la Tecnología Educativa considerando, además del contexto instruccional, el contexto socio-cultural, familiar y personal en tanto que pueden contribuir a explicar y entender el proceso de enseñanza-aprendizaje.

Prendes y Serrano (2016) apuntan que en la investigación en Tecnología Educativa ha sido frecuente confundir el uso de tecnologías como herramientas de carácter transversal y de propósito múltiple con el uso educativo de tecnologías y la investigación sobre ello. En vías de superación de los errores (u omisiones) cometidos, es preciso, a partir de los temas actuales que se abordan en la investigación en Tecnología, y que han sido recogidos recientemente, entre otros, por Cabero (2016) y Baydas, Kucuk, Yilmaz, Aydemir y Goktas (2015), plantearnos cómo se investiga en Tecnología Educativa.

Algunos meta-análisis y revisiones sistemáticas en este sentido han recogido información sobre los tipos de metodologías y enfoques metodológicos más empleados en Tecnología Educativa en los últimos años. Bulfin, Henderson, Johson y Selwyn (2014) concluyen que se ha puesto de manifiesto la tendencia a realizar estudios descriptivos y se ha detectado las limitaciones en la recopilación de los datos cuantitativos y el empleo de técnicas avanzadas de análisis de datos. En el estudio de Baydas et al (2015), se destaca que las investigaciones cuantitativas son los más frecuentes, frente a las cualitativas, y que dentro de estos los diseños cuasi-experimentales en los primeros, y el estudio de casos en los segundos, han sido los más empleados. Asimismo, los instrumentos más usados han sido los cuestionarios, en los estudios cuantitativos, y los documentos y entrevistas en los estudios cualitativos. Centrándonos en el empleo de diseños metodológicos, tanto De Benito y Salinas (2016) como Valverde (2016) abogan por las Investigaciones Basadas en el Diseño. Atendiendo al cuadrante de Pasteur propuesto por Stoke, y citado en Salinas (2012), Cabero (2016) y Valverde (2016), el enfoque basado en el diseño incluye tanto la generación de investigación "básica" como la producción y aplicación en contextos específicos, lo que lo convierten en un tipo de diseño metodológico adecuado en investigación en Tecnología Educativa.

A pesar de la escasa proliferación de los estudios cualitativos, Neuman (2014) asume que en los últimos 35 años la investigación cualitativa ha emergido como una alternativa a los estudios cuantitativos. Pero para que esto sea una realidad, sobre todo en el contexto español, se le debe atribuir valor y reconocimiento a este tipo investigaciones, más que desde el contexto investigador, desde los medios de publicación y difusión científica. En este sentido, las revistas científicas deben atender al valor de estas publicaciones desde la confiabilidad de la investigación, y no tanto desde el tamaño de la muestra. Lo habitual debería ser que 
investigaciones cuyo diseño metodológico es el estudio de casos, la fenomenología o la etnografía, entre otros, la muestra sea reducida. Por tanto, el valor de estas investigaciones no está en la representatividad de la muestra, está en el rigor metodológico que nos lleve a la confiabilidad de la investigación, que sea garantizada a través de la triangulación de las fuentes de información, la verificación de los miembros y el informe de los compañeros (Guba, 1981).

En el contexto anglosajón, son numerosas las revistas y conferencias dedicadas a la difusión de estudios cualitativos, pues la penetración de esta investigación es plena y se ha manifestado como una forma poderosa y eficaz de llevar a cabo la investigación (Neuman, 2014). En el contexto español, los procesos de investigación que conllevan innovación educativa, y por tanto, se plantean generalmente desde enfoques cualitativos y mixtos son frecuentes, pero su visibilidad en publicaciones científicas es escasa. Esto nos lleva a plantearnos si la investigación en tecnología educativa en el contexto español está siendo orquestada, sin pretenderlo, desde los criterios impuesto por las publicaciones científicas de reconocido impacto. La reflexión de la comunidad científica sobre este aspecto es necesaria, pero lo es sobre todo que comencemos a argumentar la riqueza de estas investigaciones para comprender los hechos y plantear enfoques e ideas que lo describan y le den sentido.

El número 5 de la Revista RIITE comienza con la entrevista realizada por Víctor González a Marc Prensky. En ella, el reconocido autor profundiza en los términos de Nativo digital e Inmigrante digital, concebidos como metáforas de los cambios sociales acontecidos desde la aparición de la tecnología digital, analiza su visión sobre la utilidad de la escuela actual y aporta una perspectiva crítica de la investigación en Ciencias Sociales. En este sentido, y en consonancia con lo expuesto en este editorial, Prensky considera que "TODA la investigación en Ciencias Sociales debe ser tomada sólo como una indicación de un posible hallazgo y dirección interesante, pero NO como una conclusión definitiva, válida y universal" (p. 20). Interpretamos con ello que el autor está incidiendo en la posibilidad de realizar estudios cualitativos, como alternativa a los cuantitativos, de corte experimental y cuasi-experimental, en los que las preguntas de investigación sobrepasen el fenómeno real, para interesarse por descubrir el cómo y por qué de las cosas (Neuman, 2014), buscando distintos argumentos y contrastándolos con los agentes implicados.

Lorena Casal, Carmen Fernández y Beatriz Cebreiro nos presentan, en el primer artículo del número, un análisis de la competencia del profesorado no universitario para el manejo técnico de las TIC y para su uso didáctico.

En el siguiente artículo Nuria Díez y Xavier Carrera presentan una investigación, de corte mixto, sobre la integración de Tecnologías de la Información y la Comunicación (TIC) en los procesos de enseñanza-aprendizaje de los futuros pedagogos de música. En el artículo se pone a disposición de los lectores el cuestionario usado para la investigación.

Las redes sociales en los museos de la Región de Murcia es el tema que se aborda en el artículo realizado por Antonio Domínguez e Isabel Gutiérrez. En él, desde un enfoque no experimental y un tipo de investigación descriptivo, se realiza una revisión del aprovechamiento que los centros museísticos de la Región de Murcia realizan de las redes sociales.

Por su parte, Ernesto Solano, Victoria Marín y Alba Rossi Rocha presentan los resultados de la investigación realizada en las Unidades Tecnológicas de Santander (UTS, Colombia), en relación con el nivel de conocimiento y competencia digital del personal docente adscrito a dos de las facultades de la Institución.

Mónica Marcela Sánchez Duarte y Antoni Navío nos muestran en su artículo un estudio de caso realizado en la Universidad de La Sabana (Colombia) para identificar los recursos y herramientas tecnológica más usada por los profesores, desde la perspectiva de los estudiantes. Como en artículos anteriores, el cuestionario usado en la investigación se presenta al final.

Por último, en lo que respecta a los artículos, Pilar Segura, Isabel Mํㅡㄴ Solano y $\mathrm{M}^{\mathrm{a}}$ del Mar Sánchez abordan desde una metodología mixta, el uso de las TIC en los Colegios Rurales Agrupamos en la Región de Murcia. Incluyen, asimismo, el cuestionario en el apartado de enlaces. 
El número concluye con la recensión del libro de Valverde (2015), El Proyecto de Educación Digital en un centro educativo. Guía para su elaboración y desarrollo, realizada por Pedro Antonio García Tudela.

No podemos concluir este editorial sin darle las gracias a todos los autores por sus contribuciones y por el interés manifestado por la revista RIITE, y a todos aquellos lectores que se acerquen a este número para leer las aportaciones en él incluidas. Recordamos que la Revista RIITE tiene un carácter abierto, y como tal animamos a la difusión de los contenidos de este número, compartiéndolos y comentándolos a través de nuestras redes sociales.

\section{REFERENCIAS BIBLIOGRÁFICAS}

Baydas, O., Kucuk, S., Yilmaz, R. M., Aydemir, M., y Goktas, Y. (2015). Educational technology research trends from 2002 to 2014. Scientometrics, 105(1), 709-725. DOI: 10.1007/s11192015-1693-4

Bulfin,S., Henderson, M., Johson, N. y Selwyn, N. (2014). Methodological capacity within the field of "educational technology" research: an initial investigation. British Journal of Educational Technology, 45(3), 403-414.

Cabero, J. (2016). ¿Qué debemos aprender de las pasadas investigaciones en Tecnología Educativa? Revista interuniversitaria de Investigación en Tecnología Educativa (RIITE), 0 , 22-33. DOI: 10.6018/riite/2016/256741.

Guba, E.G. (1981). Criteria for assessing the trustworthiness of naturalistic inquiries. Educational Communications and Technology Journal, 29 (2), 75-91.

Hsu, Y.-C., Hung, J.-L., y Ching, Y.-H. (2013). Trends of educational technology research: more than a decade of international research in six SSCl-indexed refereed journals. Educational Technology Research and Development, 61(4), 685-705. DOI: 10.1007/s11423-013-9290-9.

Neuman, D. (2014). Qualitative research in educational communications and technology: A brief introduction to principles and procedures. Journal Computer High Education, 26, 69-86. DOI: 10.1007/s12528-014-9078-x

Prendes, M.P y Serrano, J.L. (2016). En busca de la Tecnología Educativa: La disrupción desde los márgenes. Revista interuniversitaria de Investigación en Tecnología Educativa (RIITE), 0, 6-16. DOI: 10.6018/riite/2016/263771

Prensky, M. (2018). Entrevista a Marc Prensky. Revista interuniversitaria de Investigación en Tecnología Educativa (RIITE), 5, 21-21. DOI: 10.6018/riite/2018/354791

Valverde, J. (2016). La investigación en Tecnología Educativa y las nuevas ecologías del aprendizaje: Design-Baes Research (DBR). Revista interuniversitaria de Investigación en Tecnología Educativa (RIITE), 0, 60-73. DOI: 10.6018/riite/2016/257931 


\section{INFORMACIÓN SOBRE LA AUTORA}

\section{Isabel Ma Solano Fernández}

Universidad de Murcia

Doctora en Pedagogía por la Universidad de Murcia. Profesora Titular de Tecnología Educativa del Departamento de Didáctica y Organización Escolar de la mencionada universidad. Miembro del Grupo de investigación de Investigación de Tecnología Educativa (GITE). Ha realizado estancias de investigación en la Universidad de Cambridge y en la London Metropolitan University (Reino Unido). Entre sus líneas de investigación destacan las siguientes: Recursos multimedia audiovisuales en contexto de enseñanza formal y no formal, La Educación Infantil en contextos enriquecidos con tecnologías, Entornos Personales de Aprendizaje, Metodología de la Investigación en Tecnología Educativa y estrategias metodológicas en contextos de enseñanza formal y no formal.

Ha participado en proyectos de I+D nacionales e Internacionales relacionados con la implementación de las TIC en la sociedad y la escuela, lo que le ha permitido publicar en revistas y libros de impacto relacionados con la Tecnología Educativa.$$
\text { (c) (i) (9) }
$$

Los textos publicados en esta revista están sujetos a una licencia de Reconocimiento 4.0 España de Creative Commons. Puede copiarlos, distribuirlos, comunicarlos públicamente y hacer obras derivadas siempre que reconozca los créditos de las obras (autoría, nombre de la revista, institución editora) de la manera especificada por los autores o por la revista. La licencia completa se puede consultar en:Licencia Creative Commons Atribución-NoComercial-Compartir por igual 4.0 Internacional.
} 